\title{
Breve vistazo al impacto del cambio climático en el bosque amazónico
}

\author{
Erick J. Lavado Esteban ${ }^{1,3}$ y Marcelle São Pedro ${ }^{2,3}$ \\ Recibido: 22 julio 2020 | Aceptado: 15 febrero 2021
}

En la Amazonia, la productividad de los bosques maduros está disminuyendo de la mano con un aumento en la mortalidad de los árboles (Hubau et al., 2020; Sullivan et al., 2020). Estas alteraciones en la dinámica del bosque pueden ocasionar grandes transformaciones tanto en su estructura como en su composición (Laurance et al., 2004; Bennett et al., 2015; Sande et al., 2016). Como resultado, el efecto mitigador de estos ecosistemas sobre los impactos del cambio climático, a través de la absorción del carbono atmosférico, puede verse seriamente afectado, llevando a retroalimentaciones (feedbacks) negativas para el clima tanto en una escala local como global (Cox et al., 2000)

Esta situación de cambios negativos en los bosques es atribuida en gran parte a las consecuencias del cambio climático global y a la ocurrencia de eventos extremos como las sequías, inundaciones y olas de extremo calor (Hirabayashi et al., 2008; IPCC, 2012; Dai, 2013; Cook et al., 2014). Debido a la manera tan acelerada en que está sucediendo esta variación del clima, actualmente existe una gran preocupación sobre el destino de los diversos ecosistemas forestales (Friend et al., 2014; Trumbore et al., 2015; Gloor, 2019). No obstante, es importante señalar también que todavía hay mucha incertidumbre acerca de los reales efectos del cambio climático sobre los ecosistemas tropicales (Cox et al., 2013), lo que a su vez dificulta la predicción de cuáles serían los impactos futuros del clima sobre el planeta (Booth et al., 2012).

Temperaturas más altas, cambios en los regímenes de lluvia, y un aumento en la frecuencia e intensidad de eventos de sequía, están siendo considerados como los principales

\footnotetext{
'Facultad de Ciencias Forestales, Universidad Nacional Agraria La Molina (FCF-UNALM), Lima - Perú.

${ }^{2}$ Programa de Pós-Graduação em Ciências de Florestas Tropicais, Instituto Nacional de

Pesquisas da Amazônia (PPG-CFT / INPA), Manaus - Brasil;

elavadoesteban@gmail.com, marcellespedro.as@gmail.com

${ }^{3}$ Universidade Federal Rural do Rio de Janeiro (UFRRJ), Seropédica - Brasil.
} 
factores climáticos causantes de cambios negativos sobre la selva amazónica, siendo una mayor mortalidad y menor productividad de los bosques siempre asociados a condiciones climáticas extremas (Phillips et al., 2009, Aleixo et al., 2019). Estos efectos han llevado a lo largo del tiempo a una disminución constante en la absorción de carbono (Brienen et al., 2015) y a cambios observados en la composición de las comunidades arbóreas tanto en el nivel taxonómico - alteración de las especies - como funcional modificación en las características de las especies - (Esquivel-Muelbert et al., 2019; Costa et al., 2020). En este contexto, cabe mencionar que las condiciones en las que la lluvia ocurre en cantidades mucho menores a lo normal, comúnmente denominadas de sequías severas -como las originadas durante los años 2005, 2010 y 2015-2016-, son actualmente el foco principal de diversos estudios sobre el impacto del clima sobre el ecosistema amazónico, siendo consideradas como principales causantes de elevada mortalidad y baja productividad de los árboles en la región (Gatti et al., 2014; Feldpausch et al., 2016; Leitold et al., 2018).

El cambio climático es un, sino el mayor, desafío para la sobrevivencia no solo del ser humano sino de toda la diversidad biológica del planeta. En ese contexto, los bosques maduros de la Amazonia, que conforman el ecosistema boscoso tropical más grande del planeta, cumplen un rol esencial como mitigadores del avance del cambio climático global y de sus impactos, principalmente a través de su función como sumideros de carbono atmosférico. Sin embargo, como se evidencia en los diversos trabajos citados en este breve artículo, los árboles amazónicos -que son principales contribuidores de esta absorción- están siendo fuertemente afectados por la variación del clima, con una tendencia a lo largo de las últimas décadas a crecer menos y a morir más.

\section{LITERATURA CITADA}

Aleixo, I., Norris, D., Hemerik, L., Barbosa, A., Prata, E., Costa, F., y Poorter, L. (2019). Amazonian rainforest tree mortality driven by climate and functional traits. Nature Climate Change, 9, 384-388. doi: 10.1038/s41558-019-0458-0

Bennett, A. C., McDowell, N. G., Allen, C. D., y Anderson-Teixeira, K. J. (2015). Larger trees suffer most during drought in forests worldwide. Nature Plants, 1, 1-5. doi: 10.1038/ NPLANTS.2015.139

Booth, B. B., Jones, C. D., Collins, M., Totterdell, I. J., Cox, P. M., Sitch, S., Lloyd, J. (2012). High sensitivity of future global warming to land carbon cycle processes. Environmental Research Letters, 7, 024002. doi: $10.1088 / 1748-9326 / 7 / 2 / 024002$

Brienen, R. J. W., Phillips, O. L., Feldpausch, T. R., Gloor, E., Baker, T. R., Lloyd, J., Zagt, R. J. (2015). Long-term decline of the Amazon carbon sink. Nature, 519, 344-348. doi: $10.1038 /$ nature 14283

Cook, B. I., Smerdon, J. E., Seager, R., y Coats, S. (2014). Global warming and 21st century drying. Climate Dynamics, 43, 2607-2627. doi: $10.1007 /$ s00382-014-2075-y 
Costa, F. R. C., Zuanon, J. A. S., Baccaro, F. B., Schietti, J., Menger, J. S., Souza, J. L. P., Castilho, C. V. (2020). Effects of climate change on central Amazonian forests: a two decades synthesis of monitoring tropical biodiversity. Oecologia Australis, 24(2), 315-333. doi: $10.4257 /$ oeco.2020.2402.07

Cox, P. M., Betts, R. A., Jones, C. D., Spall, S. A., y Totterdell, I. J. (2000). Acceleration of global warming due to carbon-cycle feedbacks in a coupled climate model. Nature, 408, 184-187. doi: $10.1038 / 35041539$

Cox, P. M., Pearson, D., Booth, B. B., Friedlingstein, P., Huntingford, C., Jones, C. D., Luke, y C. M. (2013). Sensitivity of tropical carbon to climate change constrained by carbon dioxide variability. Nature, 494, 341-344. doi: 10.1038/nature11882

Dai, A. (2013). Increasing drought under global warming in observations and models. Nature Climate Change, 3, 52-58. doi: 10.1038/nclimate1633

Esquivel-Muelbert, A., Baker, T. R., Dexter, K. G., Lewis, S. L., Brienen, R. J. W., Feldpausch, T. R., Phillips, O. L. (2019). Compositional response of Amazon forests to climate change. Global Change Biology, 25(1), 39-56. doi: $10.1111 /$ gcb.14413

Feldpausch, T. R., Phillips, O. L., Brienen, R. J. W., Gloor, E., Lloyd, J., Lopez-Gonzalez, G., Vos, V. A. (2016). Amazon forest response to repeated droughts. Global Biogeochemical Cycles, 30, 964-982. doi: 10.1002/2015GB005133
Friend, A. D., Lucht, W., Rademacher, T. T., Keribin, R., Betts, R., Cadule, P., Woodward, F. I. (2014). Carbon residence time dominates uncertainty in terrestrial vegetation responses to future climate and atmospheric $\mathrm{CO} 2$. Proceedings of the National Academy of Sciences of the United States of America, 111(19), 3280-3285. doi: $10.1073 /$ pnas. 1222477110

Gatti, L. V., Gloor, M., Miller, J. B., Doughty, C. E., Malhi, Y., Domingues, L. G.,Lloyd, J. (2014). Drought sensitivity of Amazonian carbon balance revealed by atmospheric measurements. Nature, 506, 76-80. doi: $10.1038 /$ nature 12957

Gloor, E. (2019). The fate of Amazonia. Nature Climate Change, 9, 355-356. doi: 10.1038/ s41558-019-0465-1

Hirabayashi, Y., Kanae, S., Emori, S., Oki, T., y Kimoto, M. (2008). Global projections of changing risks of floods and droughts in a changing climate. Hydrological Sciences Journal, 53, 754-772. doi: 10.1623/hysj.53.4.754

Hubau, W., Lewis, S. L., Phillips, O. L., Affum-Baffoe, K., Beeckman, H., Cuní-Sanchez, A., Zemagho, L. (2020). Asynchronous carbon sink saturation in African and Amazonian tropical forests. Nature, 579, 80-87. doi: $10.1038 / \mathrm{s} 41586-020-2035-0$

IPCC. (2012). Managing the Risks of Extreme Events and Disasters to Advance Climate Change Adaptation: Special Report of the Intergovernmental Panel on Climate Change. Cambridge University Press, Cambridge, UK, and New York, NY, USA, 582 pp. 
Laurance, W. F., Oliveira, A. A., Laurance, S. G., Condit, R., Nascimento, H. E. M., SanchezThorin, A. C., Dick, C. W. (2004). Pervasive alteration of tree communities in undisturbed Amazonian forests. Nature, 428, 171175. doi: $10.1038 /$ nature 02383

Leitold, V., Morton, D. C., Longo, M., dos-Santos, M. N., Keller, M., y Scaranello, M. (2018). El Niño drought increased canopy turnover in Amazon forests. New Phytologist, 219, 959-971. doi: 10.1111/nph. 15110

Phillips, O. L., Aragao, L. E. O. C., Lewis, S. L., Fisher, J. B., Lloyd, J., Lopez-Gonzalez, G., Torres-Lezama, A. (2009). Drought Sensitivity of the Amazon Rainforest. Science, 323(5919), 1344-1347. doi: 10.1126/ science. 1164033

Sande, M. T., V. D., Arets, E. J. M. M., Peña-Claros, M., Avila, A. L., Roopsind, A., Poorter, L. (2016). Oldgrowth Neotropical forests are shifting in species and trait composition. Ecological Monographs, 86, 228-243. doi: 10.1890/15-1815.1

Sullivan, M. J. P., Lewis, S. L., AffumBaffoe, K., Castilho, C., Costa, F., Sanchez, A. C., Phillips, O. L. (2020). Long-term thermal sensitivity of Earth's tropical forests. Science, 368(6493), 869-874. doi: 10.1126/ science.aaw7578

Trumbore, S., Brando, P., y Hartmann, H. (2015). Forest health and global change. Science, 349(6250), 814-818. doi: 10.1126/ science.aac6759 